\title{
Steering Chain HIL Simulator for Steer-By-Wire Systems
}

\author{
C. Bernard*, S. Bolognani*, L. Peretti ${ }^{\dagger}$, M. Zigliotto ${ }^{\dagger}$ \\ ${ }^{*}$ Department of Electric Engineering, University of Padova, Padova, Italy \\ ${ }^{\dagger}$ Department of Engineering and Management, University of Padova, Vicenza, Italy
}

\begin{abstract}
Advanced control for automotive is one of the most promising research topic in the forthcoming decade. Actually, the long-term target is the substitution of most hydraulic car systems with their electronic counterparts. A bright example is set by the steering function, which has passed from pure mechanical to power assisted and recently to pure electrically power assisted function. The next step, the full electronic steering (Steer-By-Wire, SBW), is in progress. Definitively, it is going to substitute the mechanical connection with the steering wheel by wiretransmitted digital signals to one or more remote electric motors. Obviously, any innovative control strategy needs thorough hardware verification. At the early stages, or whether a real car prototype was not available, it is common practice to use hardware-in-the-loop (HIL) simulators, for fast control prototyping. In this frame, as proposed here, a high dynamic electric drive can virtually reproduce the real nonlinear load, represented by the steering chain and other external torque contributes. The paper presents the model details, the system architecture as well as the experimental validation of the complete HIL simulator.
\end{abstract}

\section{INTRODUCTION}

In the rush towards the inclusion of electronic systems into the vehicle, one of the forthcoming events is the electronic steering, which would use wire-transmitted digital signals, relative to driver's commands, instead of the conventional rack-pinion set. Recently, so-called Steer-By-Wire (SBW) systems receive great attention, from both car manufacturers and academic research labs.

An essential element for the successful development of new ideas is the availability of good real-time test benches ([1], [2]). Real car prototypes for the experimental test are usually available at car manufacturer's premises within the frame of a joint research program, as in the case of the present work. However, due to their cost, car prototypes are time-shared with others groups, so that it is highly recommended that they are used only for final tests. All intermediate steps, including floating to fixed point conversion, variables scaling, real-time interrupt service routine timing test and torque control tuning with real $\mathrm{AC}$ motor drive have to be accomplished meanwhile. Actually, all of these steps mean large expenditure, when performed with usual development methods. The workload comprises development of a mathematical model, as well as algorithm design and implementation, off-line simulation, and optimization. Eventually, comparison of different alternatives and their fine tuning make the overall development process extremely timeconsuming. Fast Control Prototyping (FCP) represents a smart solution, widely diffused in many research centers. Intelligent software and hardware tools relieve the control engineer from cumbersome hand coding [3]. Moreover, the hardware for FCP is normally much more powerful than the target, to release the designer from the initial uncertainties due to fine-length mathematics.

With these premises, a complete FCP test bed for the implementation of different control strategies in a SBW system has been designed and developed.

The heart of the proposed setup is the mechanical load simulator, obtained by a high dynamic PM synchronous motor, which is driven by a dedicated DSP based card. It emulates the mechanical load at the wheels of a real car during steering. Compared to [4], the new structure now includes nonlinear load simulation capability, for the virtual reproduction of effects as stiction and Coulomb friction. The drive which implements the virtual load can be coupled with different road wheel motors (RwM) under development, to test their performances under various control actions [5], [6].

The paper includes a comprehensive vehicle data set, which joins direct measurements and values obtained from a helpful car manufacturer. It thus provides the reader with the necessary information for quantitative considerations.

\section{THE STEER-By-Wire SySTEM}

Fig. 1 shows the block schematic of the complete SteerBy-Wire system, which was studied in the frame of a joint research national programme in the past two years.

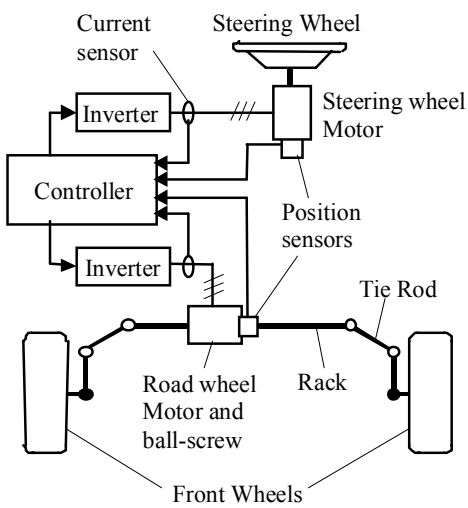

Fig. 1. The complete steering system model

This work was co-financed by the Italian National Ministry of University and Research (MIUR), and with the contribution of ELITE Programme of Texas Instruments. 
Essentially, the SBW is composed of two electrical drives that feed as many permanent magnet synchronous motors. The first, called road wheel motor (RwM), is mechanically linked to the rack, while the second (steering wheel motor, SwM) is coupled to the steering wheel, and acts as a force feedback device [5].

The test of both drives can be accomplished without the real car prototype, provided that a suitable hardware-inthe-loop load simulator is available. This has been obtained by another PMSM motor drive, coupled with the RwM, and acting as virtual mechanical load.

As shown in Fig. 1, the RwM unit imposes the desired position to the front wheels, by transmitting the motion through a ball-screw to the rack and the tie-rods. A position transducer delivers the rack position to the position regulator. In the considered configuration, the HwM is directly linked up to the steering wheel, to reduce costs and increase reliability. Another position sensor provides the steering wheel angle to the regulator.

The fundamental purpose of the control system is to regulate the torque delivered by RwM and HwM, in order to assure both correct steering angle and appropriate steering feel. The control system receives currents and position signals from the sensors, together with the vehicle speed, and gives the operating commands to the motor drives, controlling steering direction and dynamics, as well as the driver effort.

\section{ThE ViRTUAL MechanicAl LOAD}

As mentioned, a PMSM torque-controlled servo drive is used to reproduce the mechanical load constituted by both the steering chain and the reaction torque, normally addressed as self-aligning torque, which is transferred to the steering chain by the tire contact patches. In addition, the proposed HIL simulator is also able to reproduce disturbance torque, as those caused by the road ruggedness.

\section{A. Steering Chain Model}

The accurate model of the real system includes many parameters, as for example the left and right outer balljoint stiffness and the relative viscous friction and stiffness elements between the tire and the contact patch. However, to the extent of steering position control development, it has been found that the numerical values of those parameters slightly influence the dynamic behaviour, since they are mostly related to high frequency poles (approximately beyond $100 \mathrm{~Hz}$, as reported in [4]). With this in mind, the simplified steering system of Fig. 2 has been adopted in the paper.

The scheme reports both the left and the right steering chains. Due to the symmetry, it is possible to assume that the elements in the two branches have the same values, and therefore they will be recalled in the text by omitting the subscript "r", (which stands for "right") or "l" ("left").

It is worth to note that due to the simplifications in the tire behavioural model, the scheme of Fig. 2 is specific for speed greater than $30 \mathrm{~km} / \mathrm{h}$, while it could lack of accuracy if used for representing parking manoeuvres.

As said, for the C-segment car considered in this paper, the contribution of stiffness elements in the steering chain appears only around $100 \mathrm{~Hz}$, while the position control loop of a SBW requires a bandwidth of few hertz.

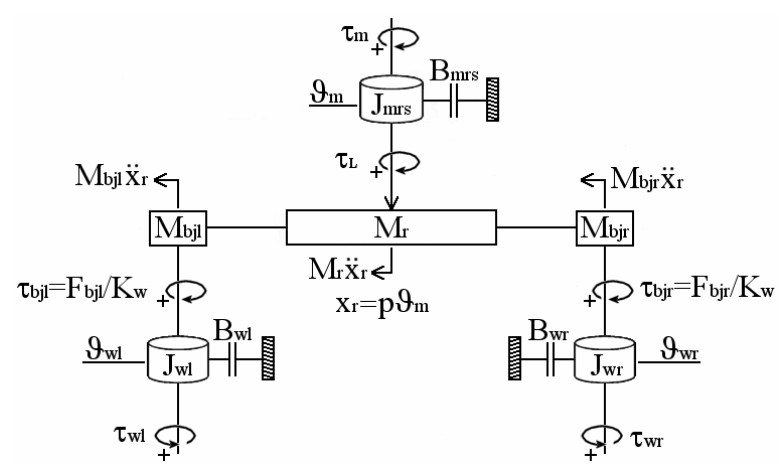

Fig. 2. The adopted steering system model.

Consequently, those elements have been neglected. Moreover, if one excludes singular operating conditions in which a particular torque is exerted on a single wheel (for example, a stone or a hole in the street), the torque twl and twr usually coincide. With the above simplifications, and by using the unified symbols for the two branches, the model equations result as follows.

$$
\begin{aligned}
& \tau_{m}=J_{m r s} \ddot{\vartheta}_{m}+B_{m r s} \dot{\vartheta}_{m}+\tau_{L} \\
& \tau_{L}=p\left(M_{r}+2 M_{b j}\right) \ddot{x}_{r}+2 p F_{b j} \\
& x_{r}=p \vartheta_{m} \\
& F_{b j}=K_{w} \tau_{b j} \\
& \vartheta_{w}=K_{m} \vartheta_{m} \\
& \tau_{b j}=J_{w} \ddot{\vartheta}_{w}+B_{w} \dot{\vartheta}_{w}
\end{aligned}
$$

and the transfer function between $\vartheta_{m}$ and $\tau_{w}$ results

$$
\frac{\tau_{L}}{\vartheta_{m}}=s^{2} p^{2}\left(M_{r}+2 M_{b j}\right)+2 K_{m}^{2}\left(J_{w} s^{2}+B_{w} s\right)
$$

All symbols are defined in Appendix $A$. Fig. 3 reports the Bode diagram of both the complete and the simplified $\vartheta_{w} / \tau_{m}$ transfer functions.

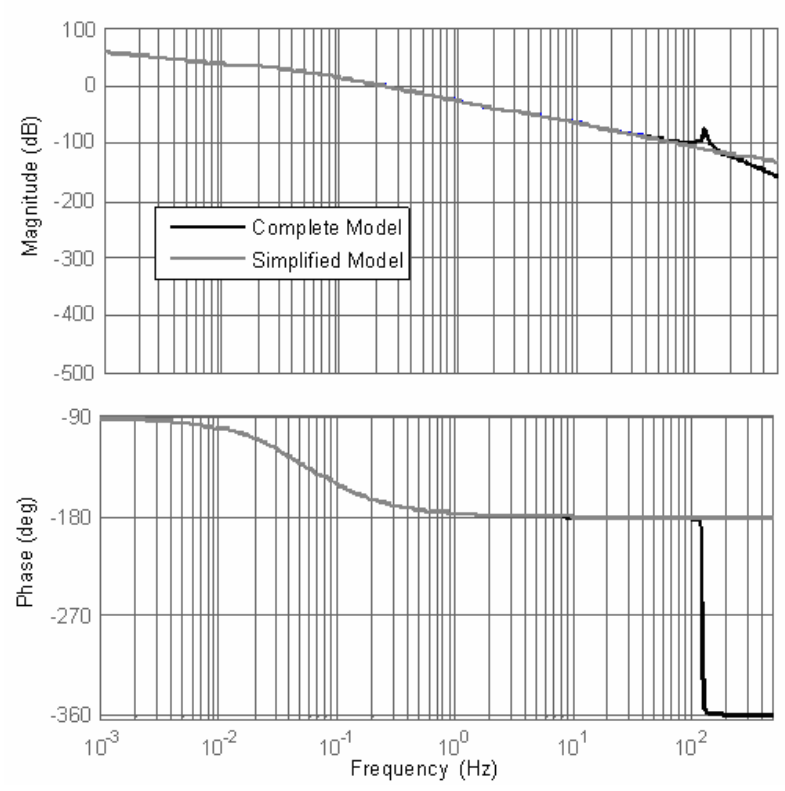

Fig. 3. Bode diagram of the transfer function $\vartheta_{w} / \tau_{m}$.

It is evident that differences appear only at high frequency, and this confirms the adopted approximations. 
The block diagram relative to expressions (1) is reported in Fig. 4. For the sake of simplicity, the steering chain model of Fig. 4 is still linear, since it does not include any non-linear friction contributes like Coulomb friction or stiction, which are surely present in the steering chain of the car. Nevertheless, as the next Section outlines, the proposed structure can be easily configured to include such non-linearity, as a substantial improvement of the results proposed in [4].

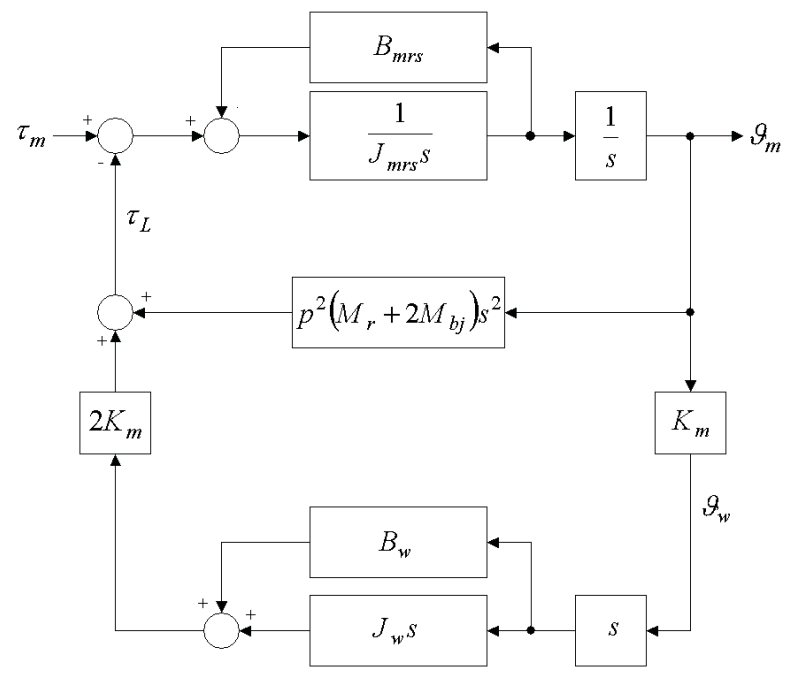

Fig. 4. Block diagram of the steering chain model.

\section{B. The Self-Aligning Torque Model}

As mentioned, the steering system is subjected to external forces, due to both random disturbances, here omitted for brevity, and a self-aligning torque $\tau_{w}$, which tends to force the wheels to a straight position with respect to the vehicle direction. Consequently, the proposed HIL simulator includes a vehicle model, used to get the relation between the aligning torque $\tau_{w}$ and the wheel position angle $\vartheta_{w}$, for any given vehicle speed $V_{g}=v_{x}+j v_{y}$.

Fig. 5 shows the two-degrees of freedom vehicle model ([7], [8]) here used as reference for the computation of the desired transfer function.
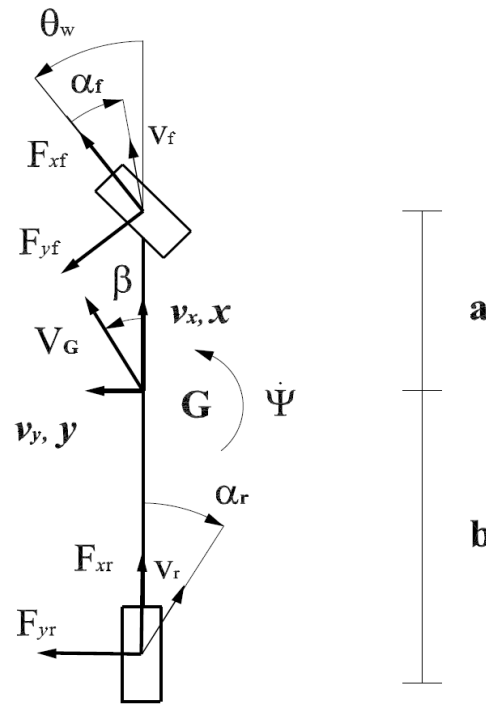

Fig. 5. Two-degrees of freedom vehicle model.
The model equations, parameterised by the vehicle speed $v_{x}$ which is assumed to be constant, are reported in (3) [9].

$$
\begin{aligned}
& m v_{x}(\dot{\beta}+\dot{\psi})=C_{f} \alpha_{f}+C_{r} \alpha_{r} \\
& J_{z} \ddot{\psi}=a C_{f} \alpha_{f}-b C_{r} \alpha_{r} \\
& \alpha_{f}=\vartheta_{w}-\beta-\dot{\psi} a / v_{x} \\
& \alpha_{r}=-\beta-\dot{\psi} b / v_{x} \\
& F_{y f}=C_{f} \alpha_{f} \\
& F_{y r}=C_{r} \alpha_{r} \\
& \tau_{w}=t_{p} F_{y f}
\end{aligned}
$$

The symbols meaning and their numerical values have been reported in Appendix B. With this model, the $\tau_{w} / \vartheta_{w}$ transfer function assumes the following form, in which the vehicle speed $v_{x}$ is to be considered as a parameter:

$$
\begin{aligned}
\frac{\tau_{w}}{\vartheta_{w}} & =\frac{n_{2} s^{2}+n_{1} s+n_{0}}{d_{2} s^{2}+d_{1} s+d_{0}} \\
n_{2} & =C_{f} t_{p} m V^{2} I_{z z} \\
n_{1} & =C_{f} t_{p} V\left(m C_{r} b^{2}+C_{r} I_{z z}\right) \\
n_{0} & =t_{p} m V^{2} C_{f} C_{r} b \\
d_{2} & =m V^{2} I_{z z} \\
d_{1} & =m V C_{f} a^{2}+m V C_{r} b^{2}+C_{f} I_{z z} V+ \\
& +C_{f} C_{r} b^{2}+C_{r} I_{z z} V \\
d_{0}= & C_{r} C_{f} a^{2}+m V^{2} C_{r} b-m V^{2} C_{f} a+ \\
& +2 C_{r} b C_{f} a
\end{aligned}
$$

The $\tau_{w} / \vartheta_{w}$ transfer function Bode diagrams, for a speed range of $30 \div 130 \mathrm{~km} / \mathrm{h}$, are shown in Fig. 6 .

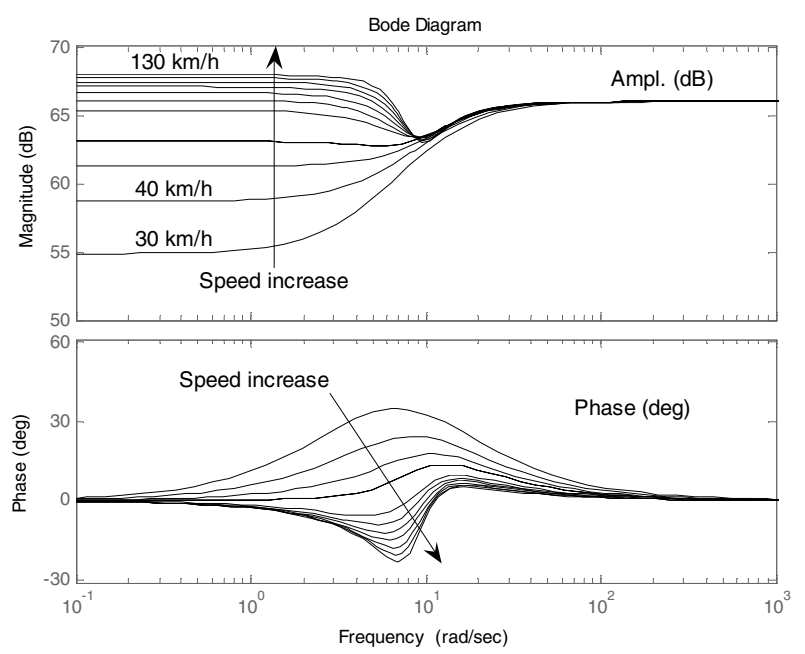

Fig. 6. Bode plot of the transfer function $\tau_{w} / \vartheta_{w}$.

Because of the aligning torque contribution, the last equation of the system (1) now becomes

$$
\tau_{b j}=J_{w} \ddot{\vartheta}_{w}+B_{w} \dot{\vartheta}_{w}+\tau_{w}
$$


In addition, the block schematic of Fig. 4, now composed of both the steering chain and the vehicle model, is modified as reported in Fig. 7.

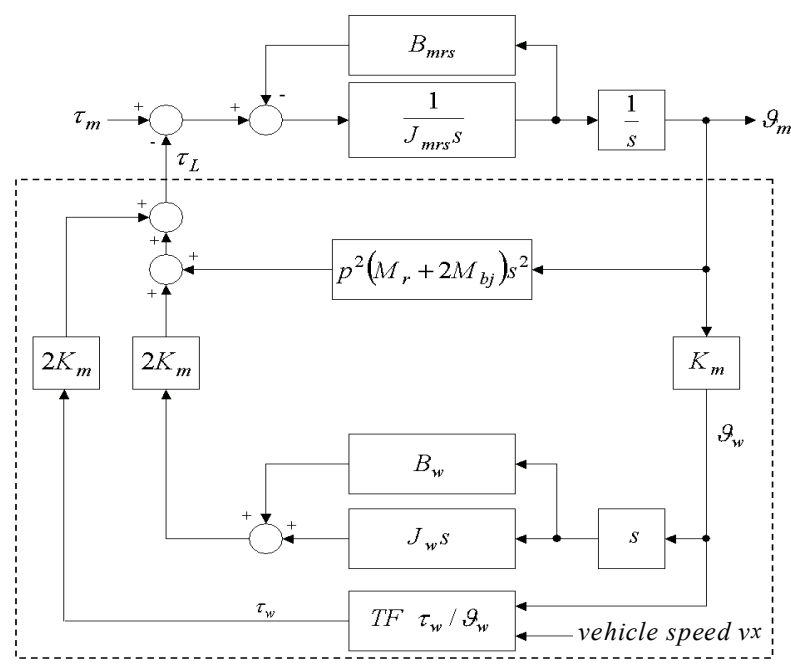

Fig. 7. Block diagram of the steering chain and aligning torque model.

\section{TEST BED IMPLEMENTATION}

The HIL simulator has been implemented on a PMSM drive, which is mechanically coupled to the road wheel motor under test.

\section{A. The HIL Simulator Model}

First of all, the mechanical coupling has been properly machined, to make its own inertia and viscous friction parameters coincide with $J_{m r s}$ and $B_{m r s}$ of Fig. 7. The HIL simulator drive is therefore committed to the reproduction of the only torque $\tau_{L}$, by means of the implementation of all the blocks inside the dotted box of Fig. 7.

The direct synthesis of the improper transfer function (2) needs the artful introduction of high-frequency poles in order to make it feasible [4]. Moreover, non-linear effects as Coulomb friction have a non-invertible transfer function $\tau / \vartheta_{m}$, which cannot be reproduced from the measurement of $\vartheta_{m}$. In this paper, a feedback approach is proposed, as outlined in Fig. 8.

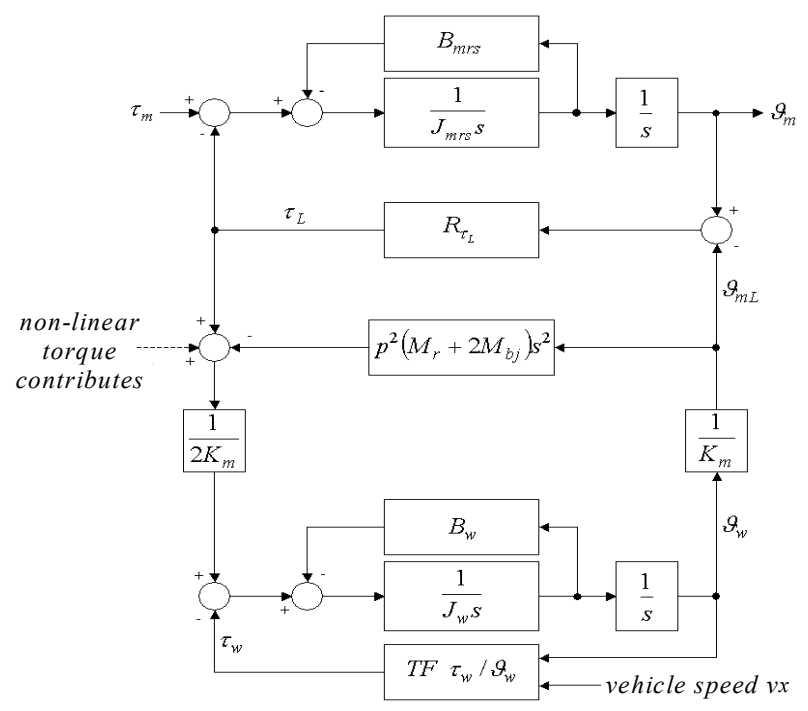

Fig. 8. Block diagram of the implemented HIL simulator.
The signal $\tau_{L}$ is the output of the regulator $R_{\tau L}$ which receives as input the difference between the measured angular position $\vartheta_{m}$ and the angular position $\vartheta_{m L}$, calculated by a DSP implementation of the model blocks fit in the dotted line of Fig. 7. The mathematical model itself, receiving as input the signal $\tau_{L}$, is now a strictly proper rational function.

The realisation is now straightforward, without any additional high frequency pole. Non-linear contributes can be added to the load torque, as depicted in Fig. 8.

\section{B. The Laboratory Prototype}

A block schematic of the test bench set up is shown in Fig. 9. It reports the RwM position control (left-hand) when the vehicle steering chain is substituted by the proposed HIL simulator (right-hand).

In particular, the RwM control system is based on a card equipped with a 33.3 ns instruction cycle TMS320C31 DSP processor, in which the position and the current control loops have been written in $\mathrm{C}$ code.

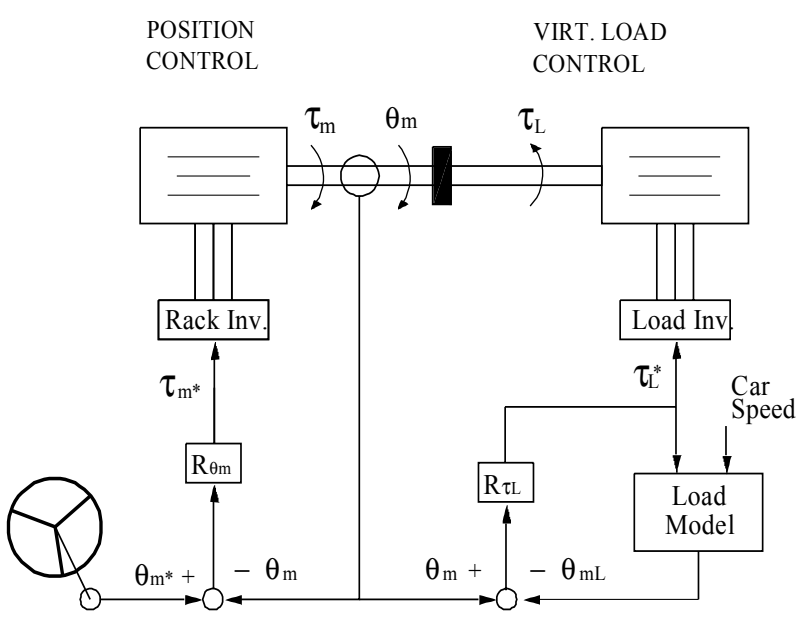

Fig. 9. Laboratory test bed set up.

The implementation of the HIL simulator has been realised by means of the Real-Time Interface (RTI) library of Matlab/Simulink, a PC plug-in card with a 40 ns instruction cycle TMS320C40 DSP processor and a current-controlled industrial drive.

The model of Fig. 8 and the I/O channel specifications of the interface card have been graphically represented in the Simulink block diagram environment, and then rapidly transferred to real-time code with the RTI, which provides the link between the hardware-in-the-loop and the Simulink itself.

The generated code delivers the reference torque signal to the industrial drive, which is considered ideal, due to the high bandwidth of its closed loop current control. This development structure is extremely flexible, and changes to HIL simulator, as well those to the control strategy, are performed very rapidly.

A conventional PID regulator well fits for $R_{\tau L}$. First design results are shown in Fig. 10 (HIL simulator openloop analysis) and Fig. 11 (HIL simulator closed-loop analysis). 


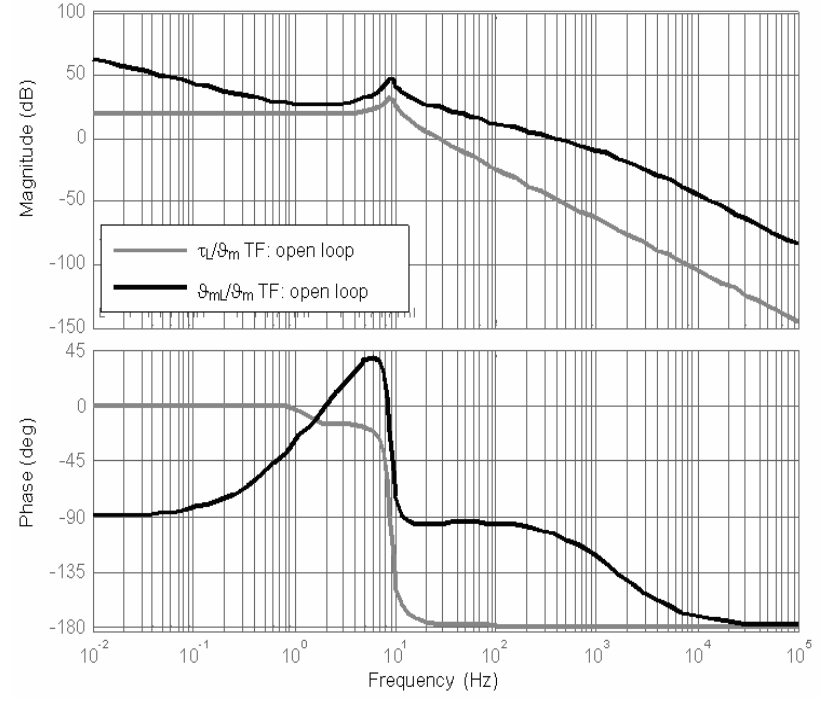

Fig. 10. HIL simulator: open loop analysis.

Bode diagrams refer to a constant vehicle speed of 72 $\mathrm{km} / \mathrm{h}$, but the control results stable for a wider range of speed, up to $250 \mathrm{~km} / \mathrm{h}$.

As expected, the closed loop response indicates that up to frequencies around $300 \mathrm{~Hz}$ the direct model of Fig. 7 is reproduced with accuracy by the proposed feedback scheme.

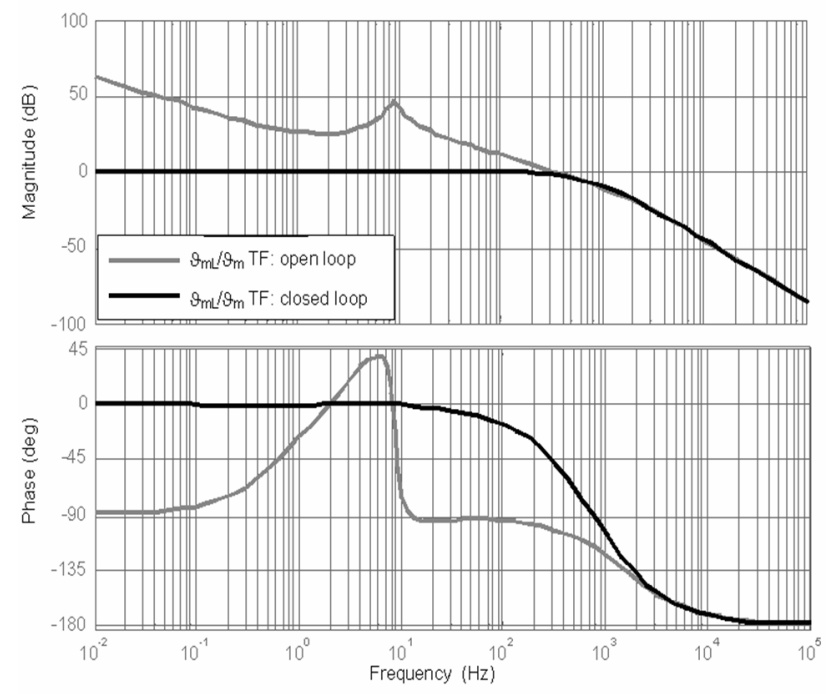

Fig. 11. HIL simulator: $\vartheta_{m L} / \vartheta_{m}$ open loop and closed loop analysis.

Fig. 12 compares the $\vartheta_{m} / \tau_{m}$ frequency responses of the ideal model of Fig. 7 and its feedback realisation of Fig. 8. In the frequency range of interest, the curves are practically overlapped at any vehicle speed. It is worth to note that the load behaves as a spring in the low frequency range (approximately below $1 \mathrm{~Hz}$ ), while it becomes an inertial load for higher frequency. It has been found that spring stiffness, resonance frequency and damping strongly depend on vehicle speed and parameters value.

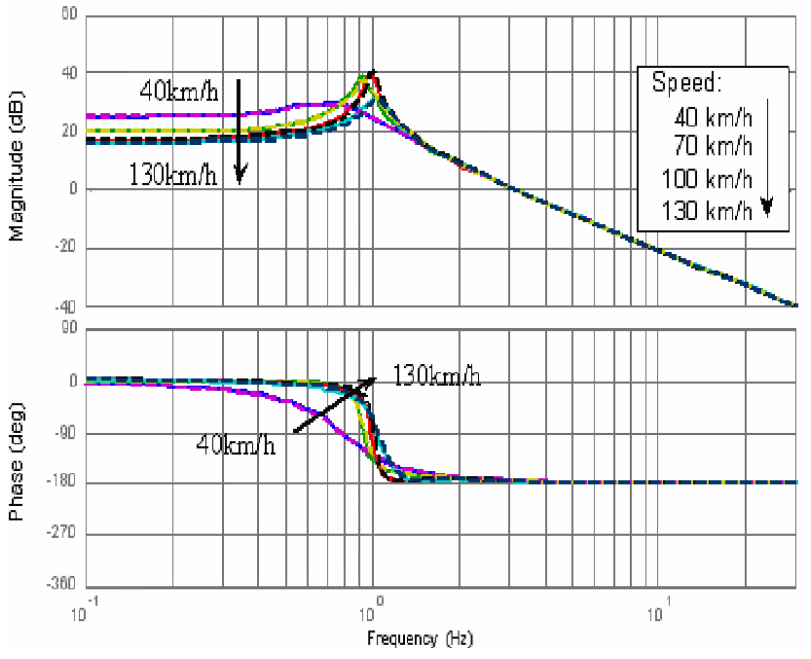

Fig. 12. HIL simulator: $\vartheta_{m} / \tau_{m}$ math model and actual implementation.

\section{Experimental Validation}

A small-signal frequency response analysis of the torque-to-position of the system of Fig. 8 has been carried out on the laboratory test bench. To this aim, a small amplitude sinusoidal input, with a frequency sweep up to $100 \mathrm{~Hz}$, was applied as $\tau_{m}$ torque reference. The resulting $\vartheta_{m}$ position outputs were collected and post-processed, to get the experimental system transfer function. The latter has been compared with that obtained mathematically from the scheme of Fig. 7. The result of the comparison is shown in Fig. 13, for a vehicle speed of $72 \mathrm{~km} / \mathrm{h}$.

The tight correspondence between the curves validates the proposed solution, confirming that the proposed feedback scheme is a smart and viable answer to the increasing demand of accurate virtual load setup, for research and development purposes. Fig. 14 reports the whole laboratory setup.

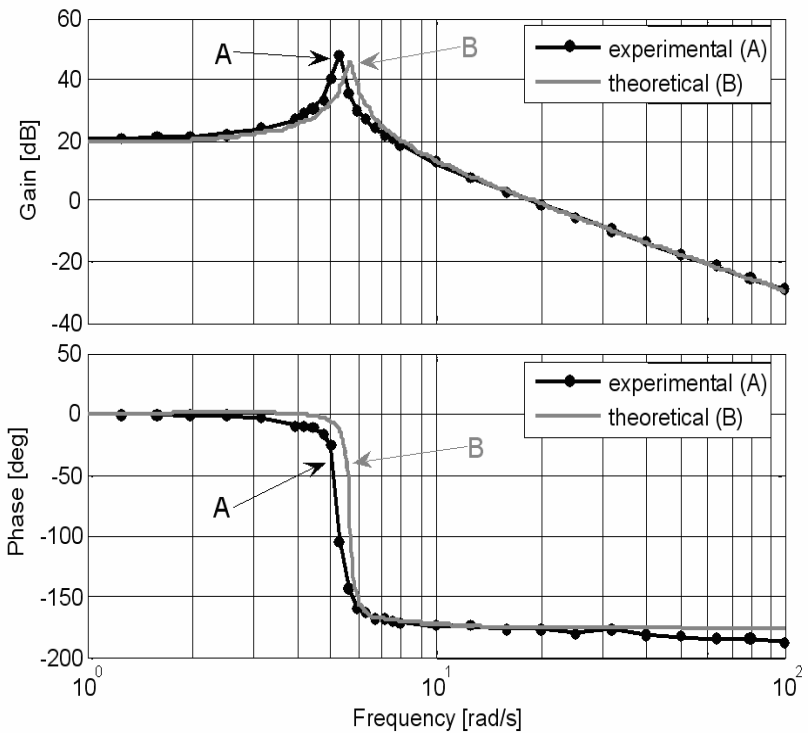

Fig. 13. Experimental and theoretical Bode diagram of the VML. 


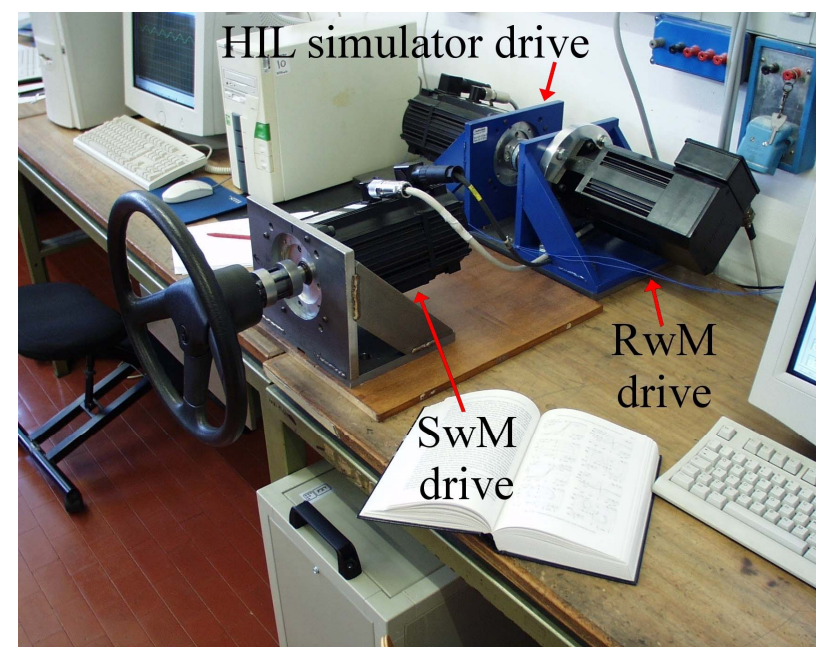

Fig. 14. The Laboratory Setup.

\section{CONCLUSIONS}

The proposed test bench has demonstrated the feasibility of a Hardware-In-the-Loop load simulator using a feedback controller approach. This represents a generalisation and enhancement of the approach presented in [4], since even non-linear contributes, which may be not negligible in a real steering system, can be modelled and experimentally reproduced, of course within the frequency bandwidth of the simulator.

The test bed also represents a precious help in the development of a complete Steer-By-Wire research project. It is expected that the concept will be exploited in future researches, with the purpose of getting a hardwarein-the-loop simulation of a variety of other critic mechanical systems.

\section{REFERENCES}

[1] T.J.Park, S.W.Oh, J.H.Jang, C.S.Han, "The Design of a Controller for the Steer-by-Wire System Using the Hardware-In-the-LoopSimulation System", SAE Techical Paper Series, 2002-01-1596, 2002.

[2] J.Ryu, H.S.Kim,"Virtual environment for developing electronic power steering and steer-by-wire systems", Conf. Proc. of IEEE/RSJ IROS, pp.1374 -1379, South Korea, 1999.

[3] R.Otterbach, T.Pöhlmann, A.Rükgauer, J.Vater, "DS1103 PPC Controller Board - Rapid Prototyping with Combined RISC and DSP Power for Motion Control“", Conf. Rec. of PCIM, Nürnberg, Germany, 1998.

[4] S.Bolognani, D.Ciscato, M.Tomasini, M.Zigliotto, "Virtual Mechanical Load Setup for Steer-by-Wire - a Case Study", Conf.Rec. IEEE-ISIE, pp.1795-1801, Dubrovnik, Croatia, 2005.

[5] A.Benedetti, N.Bianchi, S.Bolognani, M.Dai Prè, P.G.Molari, M.Tomasini, L.Tubiana, M.Zigliotto, "PM Motor Drives for Steerby-Wire Applications", Conf. Rec. of IEEE-IAS, pp. 2857-2864, Hong Kong, (2005).

[6] S.Bolognani, M.Tomasini, M.Zigliotto, "Control Design of a Steer-by-Wire System with High Performance PM Motor Drives", Proc. of IEEE-PESC, pp.1839-1844, Brasil, (2005).

[7] R.S.Sharp, M.Bettella, "Tyre shear force and moment descriptions by normalisation of parameters and the 'Magic Formula"', Vehicle System Dynamics, 39 (1), pp.27-56, 2003.

[8] H.B.Pacejka, Tyre and Vehicle Dynamics, Automotive Engineering, ISBN 0-7506-5141-5, MPG Books Ltd., 2002.

[9] S. Feick, M. Pandit, M. Zimmer, R. Uhler, Steer-by-Wire as a Mechatronic Implementation, Proc. of SAE 2000 World Congress, Detroit, 2000, SAE Paper 2000-01-0823

\section{APPENDIX $A$ - STEERING CHAIN MODEL PARAMETERS}

With reference to Fig. 2, relative to the adopted steering chain model, the symbols have the following meaning (as reported above, left and right symbols have been unified under the same nomenclature):

TABLE I.

NumericAl PARAMETERS OF THE STEERING CHAIN

\begin{tabular}{|c|c|c|}
\hline Symbol & Description & Value [Units] \\
\hline$\tau_{m}$ & $\begin{array}{l}\text { Torque supplied by the RwM to } \\
\text { the steering system }\end{array}$ & - $[\mathrm{Nm}]$ \\
\hline$\tau_{L}$ & $\begin{array}{l}\text { Torque applied onto the steering } \\
\text { system }\end{array}$ & $-[\mathrm{Nm}]$ \\
\hline$J_{m r s}$ & Rotor, nut and bearings inertia & $157,5 \cdot 10^{-6} \mathrm{~kg} \cdot \mathrm{m}^{2}$ \\
\hline$B_{m r s}$ & $\begin{array}{c}\text { Motor and ball-screw viscous } \\
\text { friction }\end{array}$ & $\frac{5 \cdot 0,05 \cdot p}{250 \cdot 10^{-3}} \frac{\mathrm{N} \cdot \mathrm{m} \cdot \mathrm{s}}{\mathrm{rad}}$ \\
\hline$\vartheta_{m}$ & RwM angular rotor position & $-[\mathrm{rad}]$ \\
\hline$p$ & $\begin{array}{l}\text { Equivalent radius (ratio between } \\
\text { linear displacement of the screw } \\
\text { and angular position of the RwM) }\end{array}$ & $\frac{5 \cdot 10^{-3}}{2 \pi} \frac{\mathrm{m}}{\mathrm{rad}}$ \\
\hline$M_{r}$ & Screw mass & $1,7 \mathrm{~kg}$ \\
\hline$M_{b j}$ & Ball-joint mass & $0,7 \mathrm{~kg}$ \\
\hline$x_{r}$ & Linear displacement of the screw & $-[\mathrm{m}]$ \\
\hline$K_{w}$ & $\begin{array}{c}\text { Tire angular displacement/ } \\
\text { ball-joint linear displacement }\end{array}$ & $\frac{30^{\circ} / 180 \cdot \pi \mathrm{rad}}{72,2 \cdot 10^{-3}} \frac{\mathrm{m}}{\mathrm{m}}$ \\
\hline$J_{w}$ & Front wheel inertia (z-axis) & $0,593 \cdot 10^{-3} \mathrm{~kg} \cdot \mathrm{m}^{2}$ \\
\hline$B_{w}$ & Front wheel joint viscous friction & $5 \frac{\mathrm{N} \cdot \mathrm{m} \cdot \mathrm{s}}{\mathrm{rad}}$ \\
\hline$\vartheta_{w}$ & Angular wheel position & - [rad] \\
\hline$\tau_{w}$ & $\begin{array}{c}\text { External torque acting on the } \\
\text { wheels }\end{array}$ & $-[\mathrm{Nm}]$ \\
\hline$K_{m}$ & $\begin{array}{l}\text { Tire angular displacement/ } \\
\text { wheel angular displacement }\end{array}$ & $p \cdot K_{w}$ \\
\hline
\end{tabular}

\section{APPENDIX $B$ - Vehicle MOdel PARAMETERS}

With reference to Fig. 5, the symbols meaning have been collected in Table II.

TABLE II.

NUMERICAL PARAMETERS OF THE VEHICLE MODEL

\begin{tabular}{|c|c|c|}
\hline Symbol & Description & Value [Units] \\
\hline$M$ & Vehicle mass & $1250[\mathrm{~kg}]$ \\
\hline$J_{z}$ & Vehicle inertia around $\mathrm{z}$ axis & $2250\left[\mathrm{~kg} \mathrm{~m}^{2}\right]$ \\
\hline$C_{f}$ & Cornering stiffness at front wheel & $69000[\mathrm{~N} / \mathrm{rad}]$ \\
\hline$C_{r}$ & Cornering stiffness at rear wheel & $\begin{array}{l}110400 \\
{[\mathrm{~N} / \mathrm{rad}]}\end{array}$ \\
\hline$\beta$ & $\begin{array}{l}\text { Side-slip angle angle (difference } \\
\text { between vehicle heading and } \\
\text { direction of velocity } V_{g} \text { ) }\end{array}$ & - [rad] \\
\hline$\alpha_{f}$ & Front wheel side-slip angle & - [rad] \\
\hline$\alpha_{r}$ & Rear wheel side-slip angle & - [rad] \\
\hline$V_{g}$ & Forward vehicle speed & $-[\mathrm{m} / \mathrm{s}]$ \\
\hline$v_{x}$ & Vehicle speed component on $\mathrm{x}$ axis & $-[\mathrm{m} / \mathrm{s}]$ \\
\hline$v_{y}$ & Vehicle speed component on y axis & $-[\mathrm{m} / \mathrm{s}]$ \\
\hline$v_{f}$ & Front wheel centroid velocity & $-[\mathrm{m} / \mathrm{s}]$ \\
\hline$v_{r}$ & Rear wheel centroid velocity & $-[\mathrm{m} / \mathrm{s}]$ \\
\hline$\dot{\psi}$ & Vehicle yaw speed & $-[\mathrm{rad} / \mathrm{s}]$ \\
\hline$l$ & Vehicle's tread & $2.546[\mathrm{~m}]$ \\
\hline $\begin{array}{l}F_{y f}, F_{x f} \\
F_{y r}, F_{x r}\end{array}$ & $\begin{array}{l}\text { Components of external road forces } \\
\text { applied on the front and rear wheels }\end{array}$ & $-[\mathrm{Nm}]$ \\
\hline$a$ & Front wheel/center of gravity distance & $0.8911[\mathrm{~m}]$ \\
\hline$b$ & Rear wheel/center of gravity distance & $1.6549[\mathrm{~m}]$ \\
\hline$\alpha_{c}$ & Caster angle & 0.0524 [rad] \\
\hline$r_{t}$ & Wheels radius & $0.2934[\mathrm{~m}]$ \\
\hline$t_{p}$ & Tire pneumatic trail & $0.0578[\mathrm{~m}]$ \\
\hline
\end{tabular}

